\title{
Efficient 3D Finite Element Modeling of a Muscle-Activated Tongue
}

\author{
Florian Vogt ${ }^{1}$, John E. Lloyd ${ }^{1}$, Stéphanie Buchaillard ${ }^{2}$, Pascal Perrier ${ }^{2}$, \\ Matthieu Chabanas ${ }^{2}$, Yohan Payan ${ }^{3}$, and Sidney S. Fels ${ }^{1}$ \\ 1 Dept. of ECE, University of British Columbia, Vancouver, Canada \\ 2 Institut de la Communication Parlée - INPG, 38031 Grenoble, France \\ 3 Laboratoire TIMC-GMCAO, Faculté de Médecine, 38700 La Tronche, France
}

\begin{abstract}
We describe our investigation of a fast 3D finite element method (FEM) for biomedical simulation of a muscle-activated human tongue. Our method uses a linear stiffness-warping scheme to achieve simulation speeds which are within a factor 10 of real-time rates at the expense of a small loss in accuracy. Muscle activations are produced by an arrangement of forces acting along selected edges of the FEM geometry. The model's dynamics are integrated using an implicit Euler formulation, which can be solved using either the conjugate gradient method or a direct sparse solver. To assess the utility of this model, we compare its accuracy against slower, but less approximate, simulations of a reference tongue model prepared using the FEM simulation package ANSYS.
\end{abstract}

\section{Introduction and Contributions}

There is an increasing need for fast, accurate simulation of the human oral pharyngeal anatomy to support research activities in medicine, dentistry, and linguistics. Applications include: the study of physiological activities (such as speech production, breathing, and swallowing), surgical and dental training, and outcome prediction for clinical procedures. Dynamic anatomical models also allow inverse modeling, in which simulation is used to estimate physiological quantities (such as muscle activation levels) which are difficult to measure directly on live subjects.

The utility of a dynamical model is highly dependent on the speed with which it can be simulated. Interactive rates are essential for haptic applications and important for researchers to interactively test the system's dynamics. Likewise, for medical practitioners, real-time interaction expands the range of possibilities for exploring "what-if" scenarios for training and pre-operative planning.

The tongue is the central organ of the oral pharyngeal anatomy and is complicated to model due to its deformable nature and intrinsic muscular activation. In this paper, we show that it is possible to rapidly calculate the dynamics of a finite element model of a muscle activated tongue (Figure 1), with reasonable accuracy, using a stiffness-warping technique such as that described in [1]. We 


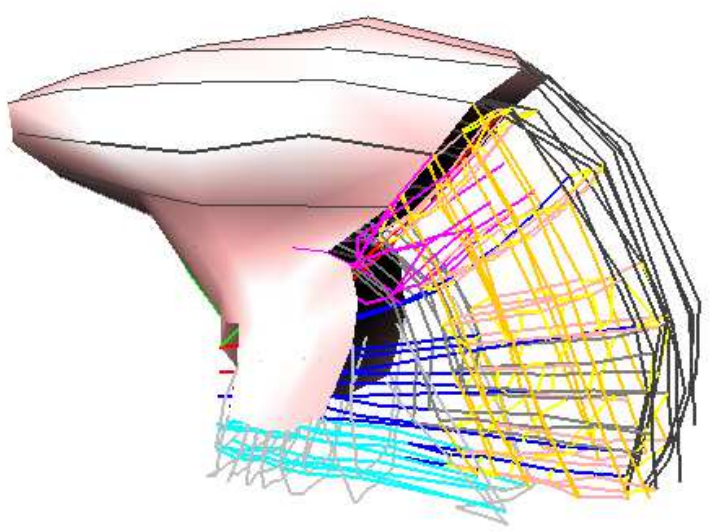

Fig. 1. Tongue model, showing its surface mesh and (in cutaway) the FEM edges corresponding to muscle fibres

build on the work of [2], who developed an FEM tongue model based on hexahedral elements and simulated it in ANSYS, which provided accurate solutions, but at very large computational cost. We test the accuracy of our faster approach by comparing it to models computed using ANSYS. The contributions of our work include:

- Combining stiffness-warping with muscle forces acting along FEM edges to create a fast model of muscle-activated tissue;

- Demonstrating that this type of model can be integrated using an implicit integrator that can be solved with a conjugate gradient solver;

- Testing the accuracy of our approach against a reported reference tongue model.

We anticipate that the techniques described here can be applied to other types of muscle-activated tissue.

This work is part of a larger project ArtiSynth [3, which is an open-source, Java-based, biomechanical simulation platform focused on the vocal tract and upper airway. One of the aims of ArtiSynth is to encourage collaboration and incremental development among scientific and medical researchers by making source code and model data easily available to the community. Source code and demos for the tongue model described in this paper can be obtained from www . artisynth. org.

\section{Related Work}

The tongue has been modeled in a wide variety of ways by various researchers. Parametric models of the tongue's shape have been developed using statistical methods 45] and spline descriptions 677. A physiological model is described in 
[8]. Dynamic models have been constructed using both mass-spring systems [9] and finite element methods [101112. A recent survey [13] describes existing methods in detail.

In addition to being efficient to simulate (ideally at interactive rates) and being validated against real measurements, an effective tongue model must provide:

- Emulation of both tissue and muscle fibre;

- Large deformations, particularly at the tip;

- Incompressible (hyperelastic) and non-linear tissue deformation.

Finite element methods provide a good solution for emulating both tissue and muscle fibre, and have a long tradition in Engineering 14 15. FEM models also provide greater stability and accuracy than mass-spring models; however, current FEM solutions 10 11 16 17 do not compute in real-time. Recent developments in the fields of physical-based animation [118] and surgical simulation [19] provide finite element algorithms which can run in real-time, albeit with less (or even unknown) accuracy, to provide plausible results even for large deformations.

Modeling the tongue's muscle activation is particularly important for simulating physiological activities such as speech and swallowing. Most current approaches to muscle tissue modeling apply Hill's non-linear spring model [20] to either mass-spring systems [9], finite elements [2118], or Cosserat models [22]. For consistency with the reference tongue model we use a muscle model based on $[12]$.

\section{Adaptation of the Reference Tongue Model Geometry}

For this work, we use the 3D FEM tongue geometry developed in 12, which is shown in Figure 1 and was developed from medical image data. This geometry contains 946 nodes, connected to form 740 hexahedral elements. These hexahedra were further subdivided into 3700 tetrahedra (using the optimal number of five tetrahedra per hexahedron) as our present implementation of the stiffnesswarping algorithm requires tetrahedral geometry.

\section{Dynamic Modeling of the Tissue and Muscle Activation}

To create a fast deformable model of the FEM tongue, we have adapted the stiffness-warping FEM approach developed by Mueller et al. 1].

Following Mueller, we use a lumped-mass FEM in which the positions of the vertices, collectively denoted by $\mathbf{x}$, obey the dynamic equation

$$
\mathbf{M} \ddot{\mathbf{x}}+\mathbf{C} \dot{\mathbf{x}}+\mathbf{K} \mathbf{x}+\mathbf{f}_{0}=\mathbf{f}_{\mathrm{ext}}+\mathbf{f}_{m}
$$

where $\mathbf{M}$ is the (diagonal) mass matrix, $\mathbf{K}$ is the stiffness matrix (based on linear Cauchy strain), $\mathbf{C}$ is the damping matrix, $\mathbf{f}_{0}$ is the rest-position stiffness forces, $\mathbf{f}_{\text {ext }}$ is the external forces, and $\mathbf{f}_{m}$ is the muscle forces (described below). This is a 
linear FEM system, except that $\mathbf{K}$ and $\mathbf{f}_{0}$ are modified at each time step to factor out the effect of elemental rotations, effectively re-referencing each element to its rest pose at the end of the previous time step. This technique, known as stiffnesswarping, allows the model to accommodate large rotational deformations with minimal volumetric distortion and a computational cost which is similar to that for linear FEMs.

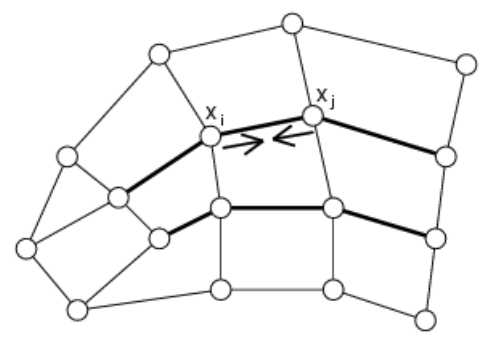

Fig. 2. Muscle forces are created by applying forces along lines between specific pairs of FEM nodes (indicated by dark lines)

To model the tongue's internal muscle activation, we designate specific collections of finite elements as muscles, each associated with an activation level (specified in Newtons). Certain FEM edges within each muscle are selected to act as "fibres", along which a uniform contractile force is exerted (Figure 2). If $i$ and $j$ are the nodes associated with such an edge, with positions $\mathbf{x}_{i}$ and $\mathbf{x}_{j}$, then the muscle forces acting on nodes $i$ and $j$ are given by $\mathbf{f}_{i j}$ and $-\mathbf{f}_{i j}$, where

$$
\mathbf{f}_{i j}=\alpha_{i j} \mathbf{u}_{i j}, \quad \mathbf{u}_{i j} \equiv \frac{\mathbf{x}_{j}-\mathbf{x}_{i}}{l_{i j}}, \quad l_{i j} \equiv\left\|\mathbf{x}_{j}-\mathbf{x}_{i}\right\|,
$$

and $\alpha_{i j}$ is the product of the muscle activation level and a weighting factor based on the masses of the nodes in the muscle. Note that this is not a Hill model [20, since the force does not depend on the edge length, and there is no damping (experiments showed that damping was adequately accounted for by the damping matrix $\mathbf{C}$ in (11)). The sum of all $\mathbf{f}_{i j}$ acting on each node produces the force term $\mathbf{f}_{m}$ in (1). In all, our model contains 11 muscles and a total of 1485 fibres. More details on the motivation for the muscle model and the selection of the muscles and fibres are given in $[17$.

Since we use a lumped-mass model, boundary conditions are effected either by applying external constraint forces to $\mathbf{f}_{\text {ext }}$ for the appropriate nodes (Lagrange multiplier approach), or by fixing certain nodes, with externally assigned positions and velocities, and then removing them from the dynamic calculation (11). The latter method is used to connect the tongue to the jaw and hyoid bone, using 117 surface nodes associated with the muscle attachment points to these structures. In the results described below, these attachment nodes are fixed rigidly. However, we are currently integrating our tongue model with the jaw/laryngeal model described in [23], and in this setting, the dynamics of the attachment nodes 
will be integrated with rigid jaw and hyoid structures, using the forces generated by the surrounding finite elements. We are also implementing a scheme to handle collisions with the teeth and soft palate, which will be implemented using soft external constraint forces applied to $\mathbf{f}_{\text {ext }}$ for the colliding nodes.

\section{Dynamic Integration}

For greater stability, we integrate (1) using an implicit Euler method. The formulation is similar to that described in [1, except that we must also account for $\partial \mathbf{f}_{m} / \partial \mathbf{x}$, since $\mathbf{f}_{m}$ is formed from the $\mathbf{f}_{i j}$, which in turn depend on the direction vectors $\mathbf{u}_{i j}$ and hence $\mathbf{x}$. Letting $h$ denote the integrator step size, $\mathbf{x}^{i}$ and $\dot{\mathbf{x}}^{i}$ the node positions and velocities at step $i$, and $\mathbf{F}_{m} \equiv \partial \mathbf{f}_{m} / \partial \mathbf{x}$, the implicit formulation is

$$
\left(\mathbf{M}+h \mathbf{C}+h^{2} \mathbf{K}-h^{2} \mathbf{F}_{m}\right) \dot{\mathbf{x}}^{i+1}=\mathbf{M} \dot{\mathbf{x}}^{i}-h\left(\mathbf{K} \mathbf{x}^{i}+\mathbf{f}_{0}-\mathbf{f}_{m}-\mathbf{f}_{\mathrm{ext}}\right)
$$

where at each step we solve for $\dot{\mathbf{x}}^{i+1}$. The computation of this solution is made easier by the fact that the matrix on the right side of (3) is symmetric positive definite (SPD):

Theorem 1. The matrix on the right side of (3) is SPD.

Proof: Without the $-h^{2} \mathbf{F}_{m}$ term, we have the original matrix in 1], which is SPD. We will show that $\mathbf{F}_{m}$ is symmetric negative semi-definite (SNSD), so that scaling it by $-h^{2}$ yields a symmetric positive semi-definite (SPSD) matrix, which when added it to the original SPD matrix yields another SPD matrix. Since $\mathbf{f}_{m}$ is the sum of force contributions for each fibre $i-j, \mathbf{F}_{m}$ is the sum of Jacobian matrices $\mathbf{F}_{i j}$ for each fibre, which are formed from $\partial \mathbf{f}_{i j} / \partial \mathbf{x}_{j}$ and $\partial \mathbf{f}_{i j} / \partial \mathbf{x}_{i}$. Referring to (2), it is fairly easy to show that

$$
\partial \mathbf{f}_{i j} / \partial \mathbf{x}_{j}=\frac{\alpha_{i j}}{l_{i j}}\left(\mathbf{I}-\mathbf{u}_{i j} \mathbf{u}_{i j}^{T}\right) \quad \text { and } \quad \partial \mathbf{f}_{i j} / \partial \mathbf{x}_{i}=-\partial \mathbf{f}_{i j} / \partial \mathbf{x}_{j}
$$

so that, ignoring zero rows and columns, $\mathbf{F}_{i j}$ has the form

$$
\mathbf{F}_{i j}=\left(\begin{array}{cc}
\partial \mathbf{f}_{i j} / \partial \mathbf{x}_{i} & \partial \mathbf{f}_{i j} / \partial \mathbf{x}_{j} \\
-\partial \mathbf{f}_{i j} / \partial \mathbf{x}_{j} & -\partial \mathbf{f}_{i j} / \partial \mathbf{x}_{i}
\end{array}\right)=-\left(\begin{array}{c}
-1 \\
1
\end{array}\right)^{T}\left(\partial \mathbf{f}_{i j} / \partial \mathbf{x}_{j}\right)\left(\begin{array}{c}
-1 \\
1
\end{array}\right) .
$$

Then, since it is possible to show that $\left(\mathbf{I}-\mathbf{u}_{i j} \mathbf{u}_{i j}^{T}\right)$, and hence $\partial \mathbf{f}_{i j} / \partial \mathbf{x}_{j}$, is SPSD, $\mathbf{F}_{i j}$ is the negative of a SPSD matrix and is hence SNSD. Finally, since $\mathbf{F}_{m}$ is formed by summing $\mathbf{F}_{i j}$, it too must be SNSD.

\section{Implementation}

Our tongue model is implemented in Java, using the modeling and numeric library support of ArtiSynth [3], which provides a framework for creating and interconnecting various kinds of dynamic and parametric models to form a complete integrated biomechanical system. 
Since execution speed is an important issue, it should be mentioned that the dynamic native code compilers (e.g., Hotspot) provided by current Java implementations usually produce execution speeds that approach compiled $\mathrm{C} / \mathrm{C}++$ code [24].

To solve the system (3), we used either the Pardiso sparse solver [25], or a conjugate gradient (CG) method; further details are given in Section 8 .

\section{Results: Accuracy}

In this section we compare the accuracy of our stiffness-warping FEM implementation (denoted as WRP) with two methods implemented using the industry standard FEM package ANSYS: (1) a linear small-deformation model (LSD), and (2) a hyperelastic Mooney-Rivlin solid model (HYP). All models used the same tetrahedral meshing described in Section 3.

The tissue elasticity parameters were obtained from the experimental work reported by [17]. For the WRP and LSD models, we used a Young's modulus of $E=6912$ and a Poisson's ratio of $\nu=0.49$. For HYP, we set $C_{1}=1152, C_{2}=$ 540 , and $\nu=0.49$. All models used a Raleigh damping (i.e., $\mathbf{C}=\alpha \mathbf{M}+\beta \mathbf{K}$ ) with $\alpha=6.22$ and $\beta=0.11$.

These models were used to simulate a set of five tasks in which a constant excitation was applied to one or more tongue muscles for 1.2 seconds and observed at a rate of $10 \mathrm{~ms}$. The tasks are named and described in Table 1 . The HYP and LSD models were computed using a variable rate ANSYS integrator, while our WRP model was computed using the single step implicit integration scheme of (3) with a fixed time step of $10 \mathrm{~ms}$.

To assess model accuracy, the deformations resulting from WRP and LSD were compared against those of the HYP (which was considered to be the most accurate and so was used as a reference). Specifically, the deformations $\mathbf{u}_{i}$ of a set of ten nodes lying on the tongue's mid-sagittal plane were compared against the reference deformations $\mathbf{u}_{r i}$ resulting from the HYP model. The deformation error $e_{i}$ at each sample point was then computed simply as

$$
e_{i}=\left\|\mathbf{u}_{r i}-\mathbf{u}_{i}\right\|
$$

Table 1. Muscle activation tasks and end-task deformation errors of these tasks (compared to HYP), resulting from the methods LSD and WRP

\begin{tabular}{|c|c|c|c|c|c|}
\hline Task & Muscle activations & \multicolumn{2}{c|}{ LSD $(\mathrm{mm})$} & \multicolumn{2}{c|}{ WRP $(\mathrm{mm})$} \\
& & $\max$ & mean & $\max$ & mean \\
\hline $\mathrm{A}$ & posterior genioglossus (2.0N) & 2.3 & 1.0 & 1.3 & 1.0 \\
$\mathrm{~B}$ & anterior genioglossus (0.5N) & 3.2 & 1.6 & 1.0 & 0.9 \\
$\mathrm{C}$ & hyoglosse $(2.0 \mathrm{~N})$ & 3.3 & 1.8 & 1.1 & 0.9 \\
$\mathrm{D}$ & transversalis $(2.0 \mathrm{~N})$ & 1.2 & 0.6 & 0.6 & 0.4 \\
$\mathrm{E}$ & inferior longitudinalis $(0.5 \mathrm{~N})$ & 1.4 & 1.1 & 0.9 & 0.8 \\
\hline
\end{tabular}




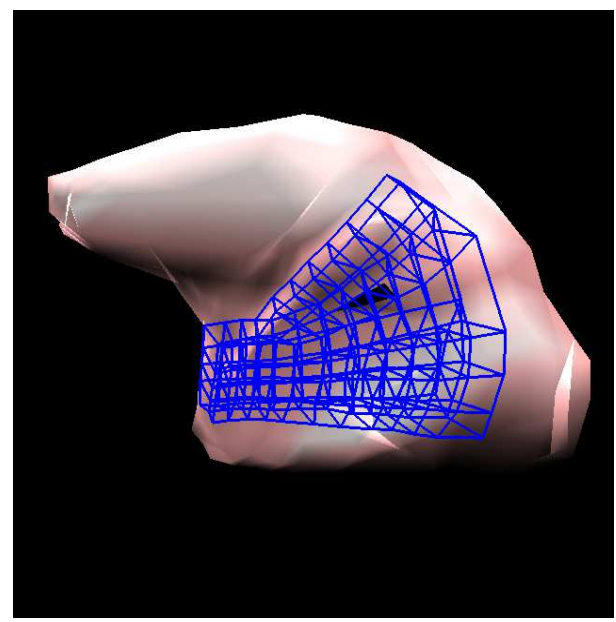

(a)

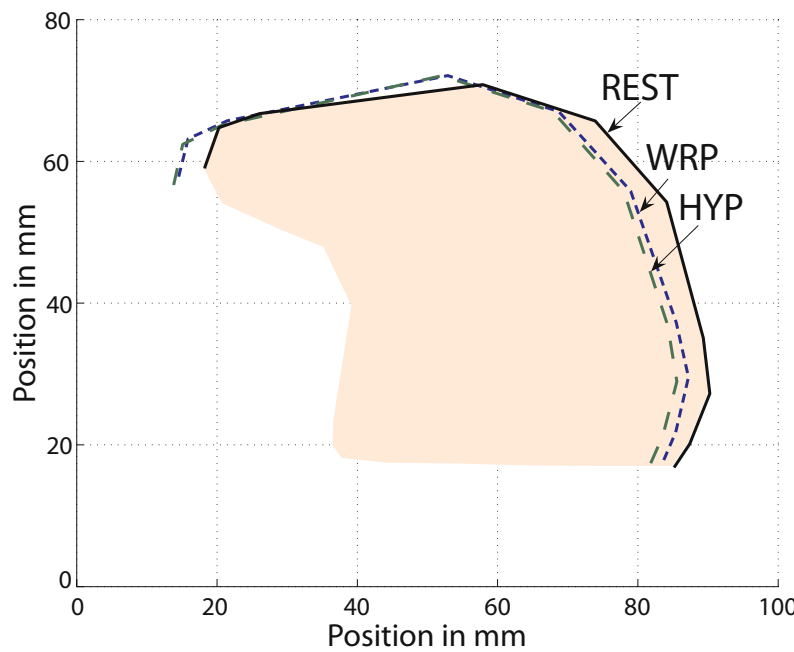

(b)

Fig. 3. (a) Tongue at rest before activation, showing the fibres of the posterior genioglossus muscle; (b) Nodes in the tongue's mid-sagittal plane before activation (REST) and after activation of Task A, as modeled by both WRP and HYP

The mean and maximum of $e_{i}$ were used to gauge the overall deformation error. Table 1 shows these values for both the WRP and LSD at the end points of each of the tasks. Figure 3(a) shows the unactivated tongue model, while 3(b) shows the mid-sagittal plane nodes before activation, and after activation for Task A as modeled by both HYP and WRP. 
Figure 3(b) suggests that the deformations produced by our stiffness-warping model do in fact adhere quite closely to those produced by the hyper-elastic reference model. This is supported more quantitatively by Table 1, where the mean error for WRP is alway within $1 \mathrm{~mm}$, and the maximum error is close to this as well, which is 2 to 3 times better than the results for LSD.

\section{Results: Speed and Stability}

Computation times for the results reported above were markedly faster for the WRP model as compared with the ANSYS LSD and HYP models, with the former requiring only about $10 \mathrm{CPU}$ seconds per simulated second, while ANSYS required about $600 \mathrm{CPU}$ seconds. All tests were run on a $2.8 \mathrm{GHz}$ Pentium IV single processor computer.

For the implicit integration step (3), we used the Pardiso spare solver [25]. We also used a conjugate gradient (CG) solver, although this was slower than Pardiso because we did not use a preconditioner and so about 300 iterations were required to achieve equivalent accuracy.

The per-step computation times were $100 \mathrm{~ms}$ and $600 \mathrm{~ms}$ for Pardiso and for unpreconditioned $\mathrm{CG}$, respectively, with $\mathrm{CG}$ requiring about $2 \mathrm{~ms}$ per iteration. About $2 \mathrm{~ms}$ of both these times was required to update the $\mathbf{K}$ and $\mathbf{f}_{0}$ terms of (1), as required by the stiffness-warping algorithm, and so this overhead is small. Using the Pardiso solver with a $10 \mathrm{~ms}$ step size resulted in a simulation speed of about 10 seconds to 1 real second.

With regard to stability, we found that our implicit solver was stable at time steps of up to $20 \mathrm{~ms}$, assuming that muscle activations were incorporated into the implicit scheme as described in Section 5. Without this, the stability limit is around $5 \mathrm{~ms}$.

\section{Conclusion and Future Work}

In this work we introduce a fast and stable finite element model and compare its performance to ANSYS FEM simulation using a previously published reference tongue model. The comparison required the adaptation of the hexahedral tessellation of the reference tongue model to a tetrahedral tessellation. Our method admits simulations speeds that are within a factor 10 of real-time, at the expense of a small loss in model accuracy.

We are considering several ways to improve the speed of our model. The first is to use a multi-threaded version of Pardiso, on a machine with several processor cores. Another possibility is to use a preconditioned CG method, which should greatly reduce the number of CG iterations required. Finally, we intend to investigate reduced coordinate approaches, along the lines of 2627 .

Continuing this work in other directions, we plan to apply these methods to other muscle groups and model the interaction with other anatomical substructures of the vocal tract. 


\section{References}

1. Mueller, M., Gross, M.: Interactive virtual materials. In: Proceedings Graphics Interface. (2004) 239-246

2. Gerard, J., Perrier, P., Payan, Y. In: 3D biomechanical tongue modelling to study speech production. Psychology Press: Sydney, Australia (In Press)

3. Fels, S., Vogt, F., van den Doel, K., Lloyd, J., Stavness, I., Vatikiotis-Bateson, E.: Artisynth: A biomechanical simulation platform for the vocal tract and upper airway. Technical Report TR-2006-10, Computer Science Dept., University of British Columbia (2006)

4. Badin, P., Bailly, G., Raybaudi, M., Segebarth, C.: A three-dimensional linear articulatory model based on mri data. In: Proceedings of the International Conference of Spoken Language (ICSLP). (1998) 14-20

5. Engwall, O.: A 3D tongue model based on MRI data. In: Proceedings of the International Conference of Spoken Language (ICSLP). (2000)

6. Stone, M., Lundberg, A.: Three-dimensional tongue surfaces from ultrasound images. In: SPIE Proc. (1996) 168-179

7. King, S.A., Parent, R.E.: A 3d parametric tongue model for animated speech. JVCA 12(3) (2001) 107-115

8. Takemoto, H.: Morphological analysis of the human tongue muscularture for threedimensional modeling. J. Sp. Lang. Hear. Res. 44 (2001) 95-107

9. Dang, J., Honda, K.: Construction and control of a physiological articulatory model. JASA 115(2) (2004) 853-870

10. Wilhelms-Tricarico, R.: Physiological modeling of speech production: methods for modeling soft-tissue articulators. JASA 97(5) (1995) 3085-98

11. Payan, Y., Perrier, P.: Synthesis of v-v sequences with a $2 \mathrm{~d}$ biomechanical tongue model controlled by the equilibrium point hypothesis. Speech Communications 22(2) (1997) 185-205

12. Gerard, J., Wilhelms-Tricarico, R., Perrier, P., Payan, Y.: A 3d dynamical biomechanical tongue model to study speech motor control. Recent Research Developments in Biomechanics 1 (2003) 49-64

13. Hiiemae, K.M., Palmer, J.B.: Tongue movements in feeding and speech. Crit Rev Oral Biol Med 14 (2003) 430-449

14. Bathe, K.J.: Finite element procedures. Prentice Hall (1996)

15. Zienkiewicz, O., Taylor, R.: The finite element method. Oxford (2000)

16. Dang, J., Honda, K.: A physiological articulatory model for simulating speech production process. JASJ 22(6) (2001) 415-425

17. Gerard, J., Ohayon, J., Luboz, V., Perrier, P., Payan, Y.: Indentation for estimating the human tongue soft tissues constitutive law: application to a $3 \mathrm{~d}$ biomechanical model to study speech motor control and pathologies of the upper airways. LNCS 3078 (2004) $77-83$

18. Teran, J., Sifakis, E., Blemker, S., Hing, N.T., V., L., C., Fedkiw, R.: Creating and simulating skeletal muscle from the visible human data set. In: IEEE TVCG (in press). (2005)

19. Cotin, S., Delingette, H., Ayache, A.: Real-time elastic deformations of soft tissues for surgery simulation. IEEE Trans. Vis. \& CG. 5(1) (1999) 62-73

20. Hill, A.: The heat of shortening and the dynamic constants of muscle. Proc Roy Soc B 126 (1938) 136-195

21. Gladilin, E., Zachow, S., Deuflhard, P., Hege., H.C.: Virtual fibers: A robust approach for muscle simulation. In: Proc MEDICON. (2001) 961-964 
22. Pai, D.K., Sueda, S., Wei., Q.: Fast physically based musculoskeletal simulation. ACM Trans Graph (2005)

23. Stavness, I., Hannam, A.G., Lloyd, J.E., Fels, S.: An integrated dynamic jaw and laryngeal model constructed from ct data. 3rd International Symposium on Biomedical Simulation (Springer LNCS) (2006)

24. Nikishkov, G.: Java performance in finite element computations. In: Proc Appl Sim \& Mod. (2003) 410

25. Schenk, O., Röllin, S., Hagemann, M.: Recent advances in sparse linear solver technology for semiconductor device simulation matrices. In: IEEE SISPAD. (2003) 103-108

26. James, D.L., Pai, D.K.: Artdefo: Accurate real time deformable objects. In: Proceedings of the International Conference on Computer Graphics and Interactive SIGGRAPH. (1999) 65-72

27. Barbic, J., James, D.L.: Real-time subspace integration for st.venant-kirchhoff deformable models. ACM Trans on Graphics 24 (2005) 982 - 990 